\title{
Influence of glistenings on the optical quality of acrylic foldable intraocular lens
}

\author{
Tetsuro Oshika, Yasuhiko Shiokawa, Shiro Amano, Kikuo Mitomo
}

\begin{abstract}
Aims-To assess the influence of glistenings on the optical quality of acrylic foldable intraocular lens.

Methods-Several degrees of glistenings in the optic were experimentally created by immersing the lens in water at $37^{\circ} \mathrm{C}$ for 48 hours and then at $25^{\circ} \mathrm{C}$ for 24 hours. Optical bench tests were carried out in water including measurements of spectral transmittance with the spectrophotometer, intensity of forward scattering using the integrating sphere photometer, modulation transfer function, and resolving power at various contrasts with and without the veiling glare light source.

Results-Glistenings of 1+ to 4+ degrees were created, among which the $4+$ glistenings seemed to be extremely intense and thought to be beyond the range of clinical settings. Clinically feasible level of glistenings, up to $3+$, did not adversely influence spectral transmittance, scattering, modulation transfer function, and resolving power at various contrasts. The $4+$ glistenings caused mild to moderate deteriorations of the optical quality of the lens.

Conclusion-The optical quality of the acrylic foldable intraocular lens is not significantly affected by the level of glistenings usually seen in the clinical setting.

(Br f Ophthalmol 2001;85:1034-1037)
\end{abstract}

Department of Ophthalmology, University of Tokyo School of Medicine,

Tokyo, Japan

T Oshika

S Amano

Department of

Electrical and

Mechanical

Engineering, Faculty

of Engineering, Chiba

University, Chiba,

Japan

Y Shiokawa

Hoya Health Care Co, Saitama, Japan

K Mitomo

Correspondence to: Tetsuro Oshika, MD Department of

Ophthalmology, University of Tokyo School of

Medicine, 7-3-1 Hongo

Bunkyo-ku Tokyo, 113-8655,

Japan

oshika-tky@umin.ac.jp

Accepted for publication 27 March 2001
The use of foldable intraocular lens (IOL) has been increasing ${ }^{12}$ as these lenses most enhance the benefits of phacoemulsification cataract surgery. Among the foldable IOLs, acrylic foldable IOLs are especially growing in popularity ${ }^{12}$ because of stable clinical results ${ }^{34}$ and a low incidence of posterior capsule opacification. $^{5-8}$ There are, however, several complications of acrylic foldable IOLs, one of which is the formation of glistenings in the optic. ${ }^{9-15}$ The glistenings are thought to be fluid accumulation in the microvoids of the optic, ${ }^{11}$ which are likely to be caused by temperature changes and not material changes. ${ }^{11}{ }^{15}$ Visual significance of glistenings is controversial $^{9}$ largely because of the lack of comprehensive optical bench testing on the lens with glistenings. In the current study, we produced an in vitro model of glistenings in the acrylic foldable IOL and evaluated its optical quality.

\section{Materials and methods}

Acrylic foldable IOLs (MA60BM, AcrySof, Alcon Surgical, Fort Worth, TX, USA) of +15.0 dioptres in the wagon wheel packaging were used in the study. The IOLs were randomly selected from a group of IOLs intended for clinical use. We used thin lenses (+15.0 dioptres) so that the incident light is focused out of the model eye in the optical bench test as described below. The optical bench tests were carried out in accordance with the ISO standard when the test methods are described in the standard (ISO 11979-2:1999(E) Optical properties and test methods).

It has been known that glistenings can be induced in vitro by warming a lens and then cooling it to room temperature. ${ }^{16}{ }^{17}$ In the current experiment, we immersed the IOLs in water at $37^{\circ} \mathrm{C}$ for 48 hours and then at $25^{\circ} \mathrm{C}$ for 24 hours. ${ }^{16}$ After the immersion, the IOLs were kept in the air and various degrees of glistenings were created by changing the duration of exposure to the air. The longer the duration of exposure to the air, the more the glistenings faded.

The scatterings of light generated by the IOL can be divided into two categoriesforward and backward scatterings. The former affects the contrast of retinal image and the latter influences the transmittance. The intensity of backward scattering was assessed by measuring spectral transmittance with a spectrophotometer (U-3210, Hitachi, Tokyo) in the spectral range of $200-800 \mathrm{~nm} .{ }^{18}$

The intensity of forward scattering was evaluated using an integrating sphere photometer (Fig 1). First, the total amount of transmitted light $(\mathrm{T})$ was measured with the rear window closed. Next, the rear window was opened and the amount of forward scattering (S) was measured. The degree of scattering (SD) is expressed as:

$$
S D(\%)=\frac{S-F}{T-F} \times 100
$$

where $\mathrm{F}$ is the intensity of stray light generated within the water cell. Using a well polished poly(methylmethacrylate) disc, the ratio of $\mathrm{F}$ to $\mathrm{T}$ was estimated to be $2.5 \%$. Since $\mathrm{F}$ is negligible in comparison with $\mathrm{T}$, equation 1 is approximated by $\mathrm{S} / \mathrm{T} \times 100-2.5$. This experiment was carried out using a $3.0 \mathrm{~mm}$ aperture.

Modulation transfer function (MTF) was measured in a model eye at a $3 \mathrm{~mm}$ aperture. ${ }^{19} 20$ The model eye was based on Gullstrand's exact schematic eye; it consisted of a front lens corresponding to the cornea, a water chamber, and a rear lens to allow for an air image. When an IOL of +15.0 dioptres was set in the water chamber, the total refractive power of the model eye approximated +53.1 dioptres. Monochromatic light of $540 \mathrm{~nm}$ and a collimating lens having a focal length of 500 $\mathrm{mm}$ were used. The test target was $10 \mu \mathrm{m}$ slit light which was projected through the eye and 

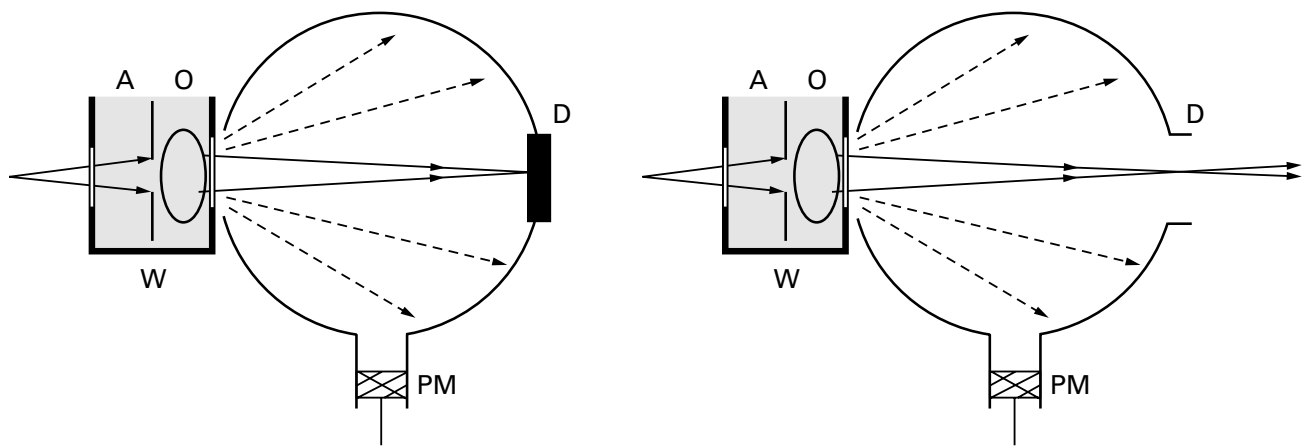

Figure 1 Schematic drawing of integrating sphere photometer to measure forward scattering. (Left) The total amount of transmitted light was measured with the rear window closed. (Right) The amount of forward scattering was measured with the rear window open.

focused on a charge coupled device image sensor. The obtained image was digitally recorded, analysed by Fourier transform, and MTF was calculated.

Resolving power of the IOL was determined using the optical bench apparatus shown in Figure 2. The IOL was immersed in the water chamber. ${ }^{21} 22 \mathrm{~A}$ variable contrast acuity chart ${ }^{23}$ was used as the resolution target to assess the resolving power at five different contrasts - that is, $13,30,45,60$, and $83 \%$. For each contrast, the chart theoretically allows the minimum and maximum resolving power of 4 and 60 cycles per degree (equivalent to 20/150 and 20/10 of Snellen chart visual acuity), respectively. As the veiling glare light source, six fluorescent lamps of $20 \mathrm{~W}$ were placed by the contrast acuity chart. The chart luminance and veiling glare illuminance on the aperture plane were set according to the conditions of MCT 8000 (Vistech Consultants, Inc, Dayton, $\mathrm{OH}$, USA). ${ }^{24}$ The chart images focused in the model eye were magnified by the microscope, which were evaluated by three independent examiners and the results were averaged. The parameter of glare sensitivity was then calculated by subtracting the resolving power with glare light on $\left(\mathrm{R}_{\mathrm{on}}\right)$ from that without glare light $\left(R_{\text {off }}\right)$. Values were then summed up for five different contrast levels.

Glare sensitivity $=\sum\left(\log R_{\text {off }}-\log R_{o n}\right)$

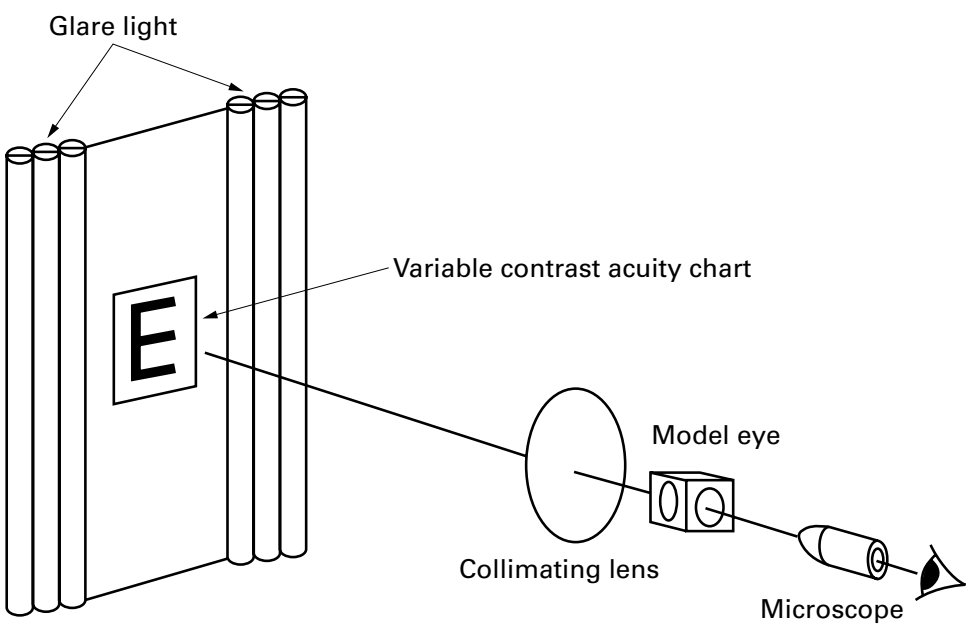

Figure 2 Optical bench apparatus to measure resolving power of the lens.

\section{Results}

Several degrees of glistenings were created, which were graded from $1+$ to $4+$ as shown in Figure 3. The evaluation was performed on photographs. ${ }^{1625}$ The $1+$ to $3+$ glistenings appeared to be compatible with the clinically feasible degree of glistenings, but the $4+$ level was extremely intense and thought to be beyond the range of clinical settings. ${ }^{16}{ }^{25}$ Since the $1+$ glistenings were rather quick to disappear, this lens could not undergo the following experimental procedures under a constant condition. The $2+$ to $4+$ IOLs remained stable during the optical bench tests, and thus the data of these and the control lenses are shown below. One representative lens was used for each level of glistenings, and measurements were repeated twice. When the results of two measurements varied, third measurement was carried out.

The results of spectral transmittance measurements are shown in Figure 4. The IOLs with $2+$ and $3+$ glistenings exhibited spectral transmittance similar to that of the control lens, indicating that clinical level of glistenings do not adversely affect the light transmittance. The extremely severe glistenings $(4+)$ decreased spectral transmittance especially at longer wavelength.

The degrees of scattering calculated by equation 1 were $3.9 \%$ for the control lens, $5.9 \%$ for the lens with $2+$ glistenings, $11.1 \%$

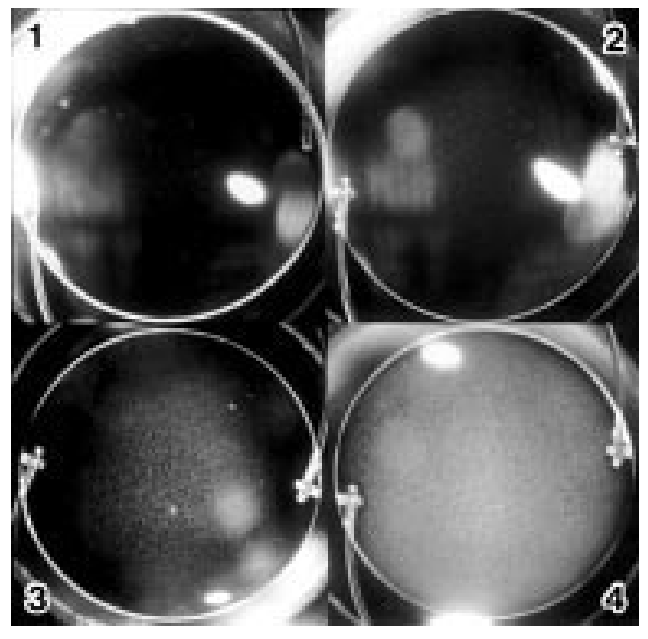

Figure 3 Slit lamp photograph of glistenings ranging from $1+$ to $4+$. 


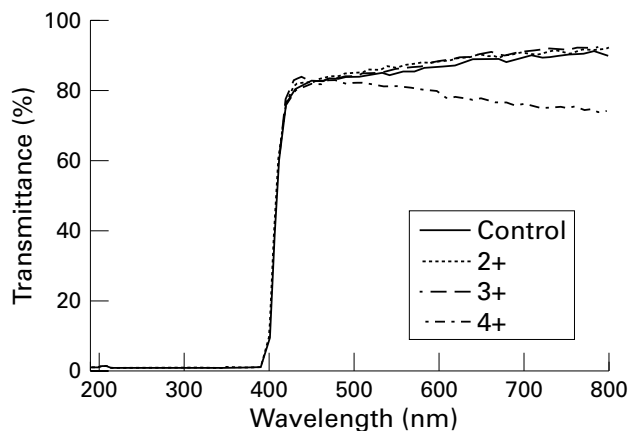

Figure 4 Spectral transmittance.

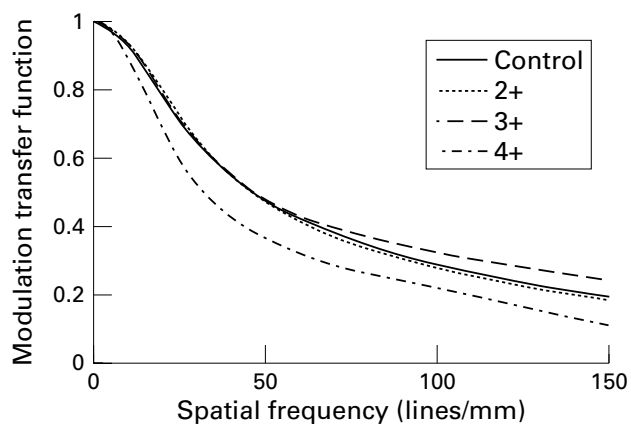

Figure 5 Modulation transfer function.

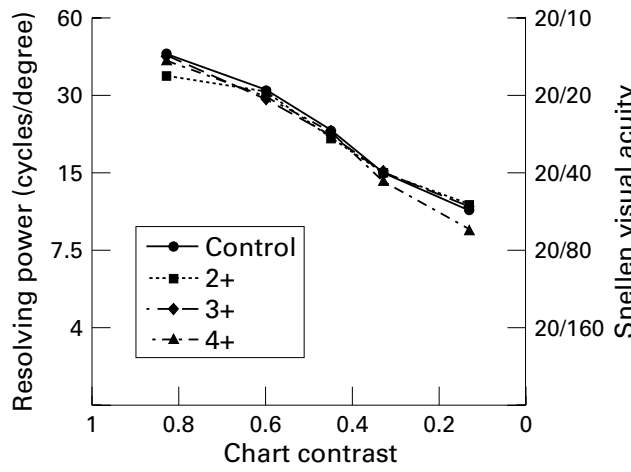

Figure 6 Resolving power at various contrasts without veiling glare light.

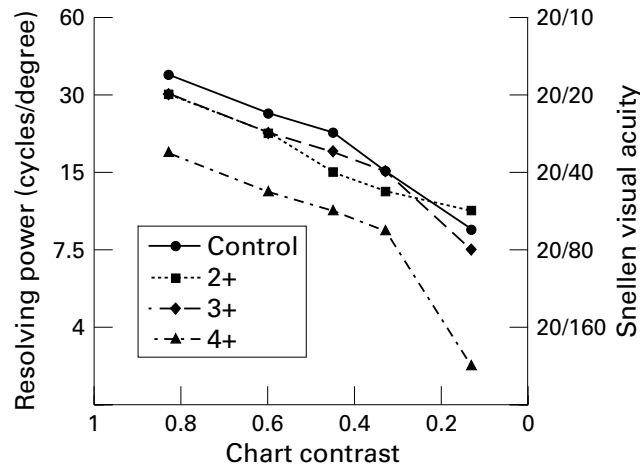

Figure 7 Resolving power at various contrasts with veiling glare light on.

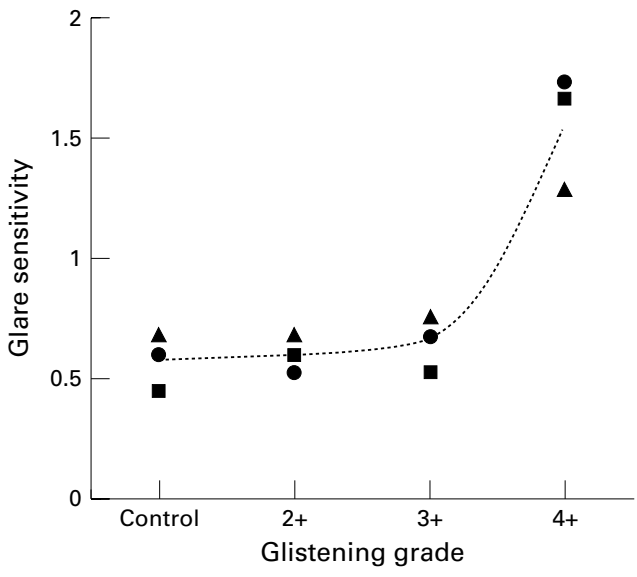

Figure 8 Glare sensitivity index evaluated by three examiners. The line indicates the average.

for $3+$ glistenings, and $21.7 \%$ for $4+$ glistenings. The measurement results of MTF are shown in Figure 5. The glistenings up to $3+$ did not affect MTF, while 4+ glistenings caused slight deterioration of MTF.

Resolving power of the IOL was evaluated in relation to the chart contrast and the presence of veiling glare light. When the glare light was off, there was no significant influence of glistenings on the resolving power (Fig 6). When the glare light was turned on, resolving power of IOL with 4+ glistenings significantly deteriorated especially at lower contrast (Fig 7). Resolving power was unaffected when the glistenings were $3+$ or less. The parameter of glare sensitivity calculated by equation 2 is shown in Figure 8. The glare sensitivity did not change significantly up to $3+$ glistenings, but deteriorated significantly with $4+$ glistenings.

\section{Discussion}

In the current study, we experimentally induced glistening particles in the optic of acrylic foldable IOLs, ranging in degree from $1+$ to $4+$. Although the $1+$ glistenings could be photographed (Fig 3), their instability made the optical bench test on this lens impossible. Nevertheless, judging from the experimental results of the $2+$ glistening lens, data of the $1+$ glistening IOL should have been similar to those of the control lens.

In the measurements of spectral transmittance, the IOLs with clinically compatible level of glistenings $(2+$ and $3+)$ showed results similar to those of the control lens. On the other hand, very severe glistenings (4+) deteriorated spectral transmittance especially at longer wavelength. The diameter of glistening particles has been reported to be approximately 10-20 $\mu \mathrm{m} .{ }^{16}$ Theoretically, this size of particles induce Mie scattering, where diffraction plays a significant part. In general, the longer the wavelength of light the larger the diffraction angle becomes. When the diffraction angle is large, a greater portion of scattering attributable to diffraction is lost from transmission, resulting in lower transmittance. The 4+ glistenings, however, are extremely intense and thought to be beyond the range of clinical settings, and thus the clinical significance of this finding is unclear. 
Other optical parameters tested in the current study were scattering, MTF, and resolving power at various contrasts with and without the veiling glare light source. As shown in the results, the glistenings up to $3+$ had little influence on these parameters. In practice, glistenings are frequently seen in acrylic foldable IOL after several months postoperatively, ${ }^{16}$ but very few cases have led to significant clinical consequences. ${ }^{17}$ The data obtained here objectively confirm such clinical observations.

It has been known that the glistenings only occur under fluid, representing an influx of aqueous humour into the optic and not the degeneration of materials. ${ }^{17}$ This theory has been supported by the observation with optical and atomic microscopy. ${ }^{15}$ Moreover, the formation of glistenings is not limited to acrylic foldable IOL, but can be seen with poly(methylmethacrylate) and silicone IOLs. ${ }^{17}{ }^{26}$ As in acrylic foldable IOL, glistenings in these latter biomaterials have not been shown to cause any adverse clinical sequelae. The discoloration of silicone IOLs was also related to water vapour absorption within the IOL, having a brownish appearance from light scattering. ${ }^{27}$ These artefacts had no adverse effect on the patients' visual acuity. ${ }^{28}$ Thus, we conclude that the clinically compatible level of glistening formation in the optic of IOL is principally a cosmetic phenomenon, but not an optical incident.

Proprietary interests: none.

1 Leaming DV. Practice styles and preferences of ASCRS members-1999 survey. $\mathcal{f}$ Cataract Refract Surg 2000;26:913-21.

2 Oshika T, Amano S, Araie M, et al. Current trends in cataract and refractive surgery in Japan. 1998 Survey. $\mathcal{F p n ~}^{\mathcal{F}}$ Ophthalmol 2000;44:268-76.

3 Oshika T, Suzuki Y, Kizaki H, et al. Two year clinical study of a soft acrylic intraocular lens. I Cataract Refract Surg 1996;22:104-9.

4 Mengual E, Garcia J, Elvira JC, et al. Clinical results of AcrySof intraocular lens implantation. $f$ Cataract Refract Surg 1998;24:114-7.

5 Ursell PG, Spalton DJ, Pande MV, et al. Relationship between intraocular lens biomaterials and posterior capsule opacification. F Cataract Refract Surg 1998;24:352-60.

6 Hollick EJ, Spalton DJ, Ursell PG, et al. The effect of polymethylmethacrylate, silicone, and polyacrylic intraocupolymethylmethacrylate, silicone, and polyacrylic intraocu-
lar lenses on posterior capsular opacification 3 years after cataract surgery, Ophthalmology 1999;106:49-54; discussion 54-5.

7 Hollick EJ, Spalton DJ, Ursell PG, et al. Lens epithelial cell regression on the posterior capsule with different intraocular lens materials. Br f Ophthalmol 1998;82:1182-8.

8 Oner FH, Gunenc U, Ferliel ST. Posterior capsule opacification after phacoemulsification: foldable acrylic versus poly(methyl methacrylate) intraocular lenses. 7 versus poly(methyl methacrylate) intract Refract Surg 2000;26:722-6.

9 Dhaliwal DK, Mamalis N, Olson RJ, et al. Visual significance of glistenings seen in the AcrySof intraocular lens. F Cataract Refract Surg 1996;22:452-7.

10 Chehade M, Elder MJ. Intraocular lens materials and styles: a review. Aust NZ F Ophthalmol 1997;25:255-63.

11 Omar O, Pirayesh A, Mamalis N, et al. In vitro analysis of AcrySof intraocular lens glistenings in AcryPak and Wagon Wheel packaging. f Cataract Refract Surg 1998;24:107-13.

12 Arshinoff S. Does a tight capsular bag cause glistenings? $f$ Cataract Refract Surg 1998;24:6.

13 Peetermans E, Hennekes R. Long-term results of wagon wheel packed acrylic intra-ocular lenses (AcrySof). Bull Soc Belge Ophtalmol 1999;271:45-8.

$14 \mathrm{Klos} \mathrm{KM}$, Richter R, Schnaudigel O, et al. Image analysis of implanted rigid and foldable intraocular lenses in human eyes using Scheimpflug photography. Ophthalmic Res 1999; 31:130-3.

15 Dogru M, Tetsumoto K, Tagami Y, et al. Optical and atomic force microscopy of an explanted AcrySof intraocular lens with glistenings. F Cataract Refract Surg 2000;26:571-5.

16 Miyata A, Suzuki K, Boku C, et al. Glistening particles on the implanted acrylic intraocular lens. Fpn f Clin Ophthalmol (Fapanese) 1997;51:729-32.

17 Apple DJ, Auffarth GU, Peng Q, et al. Hydrophobic acrylic intraocular lenses. In: Apple DJ, Auffarth GU, Peng Q, Visessook N, eds. Foldable intraocular lenses. Evolution, clinicopathologic correlation, and complications. Ch 5. Thorofare, NJ: Slack, 2000:103-22.

18 Mainster MA. Spectral transmittance of intraocular lenses and retinal damage from intense light sources. Am $\mathcal{F}$ Ophthalmol 1978;85:167-70.

19 Portney V. Optical testing and inspection methodology for modern intraocular lenses. F Cataract Refract Surg 1992;18: 607-13.

20 Oshika T, Shiokawa Y. Effect of folding on the optical quality of soft acrylic intraocular lenses. F Cataract Refract Surg 1996;22(Suppl 2):1360-4.

21 Holladay JT, Ting AC, Koester CJ, et al. Intraocular lens resolution in air and water. $\mathcal{F}$ Cataract Refract Surg 1987;13: 511-7.

22 Holladay JT, Ting AC, Koester CJ, et al. Silicone intraocular lens resolution in air and in water. $\mathcal{F}$ Cataract Refract Surg 1988;14:657-9.

23 Wang GJ, Pomerantzeff O. A new set of variable-contrast visual acuity charts. Optom Vis Sci 1991;68:34-40.

24 Pfoff DS, Werner JS. Effect of cataract surgery on contrast sensitivity and glare in patients with $20 / 50$ or better Snellen acuity. 7 Cataract Refract Surg 1994;20:620-5.

25 Minami H, Torii K, Hiroi K, et al. Glistening of the acrylic intraocular lenses. Fpn $\mathcal{f}$ Clin Ophthalmol (fapanese) 1999;53:991-4

26 Ballin N. Glistenings in injection-molded lens. $\mathcal{F} \mathrm{Am}$ Intraocul Implant Soc 1984;10:473.

27 Milauskas AT. Silicone intraocular lens discoloration in humans. Arch Ophthalmol 1991;109:913.

28 Koch DD, Heit LE. Discoloration of silicone intraocular lenses. Arch Ophthalmol 1992;110:319-30. 